1 V. V. Baron u. E. M. Savitskiy, Zh. Neorgan. Khim. 6, 182 [1961].

2 Ch. E. Lundin u. A. S. Yamamoto, Trans. Aime 236, 863 [1966].

3 C. R. McKinsey u. G. M. Faulring, Acta Cryst. 12, 701 [1959].

${ }^{4}$ P. J. Brown u. J. B. Forsyth, Acta Cryst. 14, 362 [1961].

5 K. P. Gupta, Trans. AIME 221, 1047 [1961].

${ }^{6}$ E. Corenzwit, J. Phys. Chem. Sol. 9, 93 [1959].

7 J. Johnston, University of California, Berkeley; Lawrence Rad. Lab. UCRL-11390 (1964).

8 A. Müller, Z. Naturforsch. 25 a, 1659 [1970].

\title{
Elastic Constants of Nematic Liquid Crystals
}

\author{
II. Splay $\left(k_{11}\right)$ and Bend $\left(k_{33}\right)$ Elastic Constants of 4,4'-Di(n-alkoxy)azoxybenzene for $n=1$ to 6
}

HANS GRULER

Institut für Angewandte Festkörperphysik der Fraunhofer-Gesellschaft, Freiburg i. Br.

(Z. Naturforsch. 28 a, $474-483$ [1973] ; received 7 December 1972)

In this paper the splay $k_{11}$ and bend $k_{33}$ elastic constants for the homologous series $4,4^{\prime}$ - $d i$ (nalkoxy) azoxybenzene (from methyloxy to hexyloxy) were measured as a function of temperature by studying the deformation of the liquid crystal in a magnetic field. The values of these elastic constants $\left(10^{-6}\right.$ to $10^{-5}$ dyne) all decrease with increasing temperature. The reduced elastic constants $C_{11}$ and $C_{33}$ which were derived by Saupe and which take into consideration the effect of temperature on the order parameter and on the mole volume should be independent of temperature if there are no changes in short range order.

In the region of the isotropic transition temperature the reduced elastic constants of the homologous series show a general increase with increasing alkoxy chain length. This trend can be explained by the increasing molecular length. There is still a strong alternation superimposed upon this trend, however, which can not be due to the alternation of the anisotropic polarizabilities.

To first approximation, the elastic constant ratios for the homologous series can be summarized on a single universal curve. The temperature behavior of the elastic constants can be divided into four groups, each having its own characteristic short range order structure.

Region IV, which is near the smectic phase transition, is characterized by cybotactic groups which exist in the nematic phase and which have a smectic like structure. $C_{33}$ as well as $C_{11}$ show a very strong increase in this region as the smectic $C$ phase is approached. This abnormal temperature behavior can be explained as a pretransitional effect because in the smectic $\mathrm{C}$ phase $C_{33}$ and $C_{11}$ must be quite large because of the planar structure where the molecules are tilted with respect to the plane. In a smectic A phase where the molecules are perpendicular to the plane a pretransitional effect occurs only in $C_{33}$ as Cheung and Meyer have shown.

In region III $C_{33} / C_{11} \sim 1$ and this ratio is temperature independent. This behavior is in agreement with our model calculation if the steric units contain only a few molecules and are approxi mately spherical and are arranged to form a planar structure.

In region II $C_{33} / C_{11} \sim 2$ and $C_{11}$ and $C_{33}$ are both independent of temperature. The assumptions made for region III are also valid here except that the steric groups in region II are not arranged in a planar configuration. This assumption is in agreement with the model calculation and has been verified through $\mathrm{x}$-ray experiments.

In region $\mathrm{I}$, which borders upon the isotropic phase, $C_{11}$ is nearly independent of temperature whereas $C_{33}$ becomes smaller with inceasing temperature. The model calculation shows that $C_{33}$ is more sensitive to structural changes than $C_{\mathbf{1 1}}$. The abnormal temperature behavior of the mole volume in this region is an indication that changes in the short range order structure are occurring.

\section{Introduction}

The elastic distortions of liquid crystals have been the subject of many studies. Oseen proposed that the distortional energy density in a liquid crystal could be described by a sum of specific curvature distortions of the local optical axis where every curvature is associated with its own elastic constant ${ }^{1}$. Oseen's predictions took on a more practical aspect after Zocher interpreted the distortions

Reprint requests to H. Gruler, Gordon McKay Laboratory, Harvard University, Cambridge, Mass. 02138, USA. in a nematic liquid crystal as being of a continuous nature, taking place throughout the bulk rather than occurring in swarms ${ }^{2}$. Frank reformulated parts of Oseen's theory and stimulated further interest in the elastic properties of liquid crystals ${ }^{3}$. Recently, using both a molecular and a phenomenological approach, Nehring and SAUPE have brought together these earlier studies ${ }^{4}$. JÄHNIG and SCHMidT ${ }^{5}$ as well as Martin, Parodi, and Pershan ${ }^{6}$ have given more general consideration to the elastic properties of liquid crystals. 
The molecular statistical theory of Maier and Saupe shows that these elastic constants should be very sensitive to short range order $7,8,9$, precise measurements of elastic constants are important if any short range order models for liquid crystals are to be experimentally verified.

In the following study the bend and splay elastic constants for six members of the 4,4'-di(n-alkoxy)azoxybenzene series have been measured as a function of temperature. A smectic C phase appears in the higher homologs of this series and our elastic constant measurements indicate a considerable change in short range order near the transition for these compounds.

\section{II.A A Comparison of the Elastic Constant Values Given in the Literature for $4,4^{\prime}-\mathrm{Di}($ methyloxy)- azoxybenzene}

The elastic constants of nematic liquid crystals can be determined by a variety of methods. The classical techniques were recently described by Gruler, Scheffer, and Meier ${ }^{10}$. Saupe ${ }^{9}$ compared the elastic constants $k_{11}$ and $k_{33}$ of methyloxyazooxybenzene (PAA) obtained using the threshold field values of the normal deformation (Sect. II.A of Ref. ${ }^{10}$ ) with those obtained from a detailed analysis of the optical phase difference change curve as a function of field and found different values for these elastic constants by these two different methods. The results of both measuring methods are shown in Fig. 1 where the ratio of the elastic con-

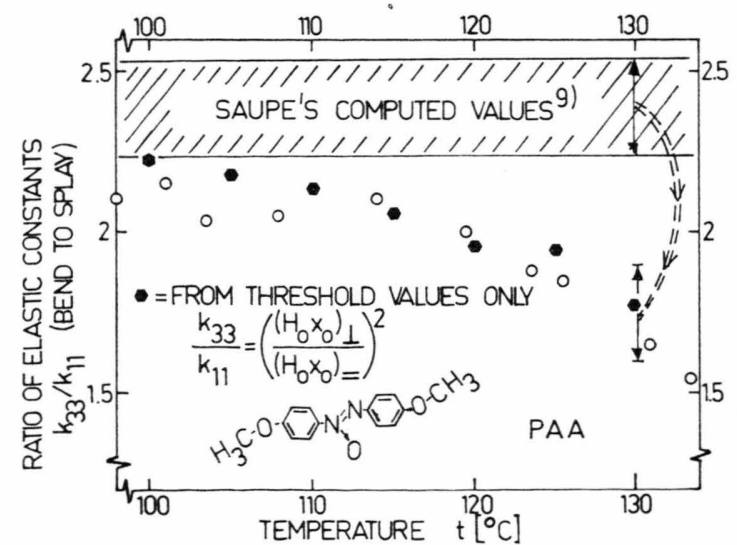

Fig. 1. Comparison of the measuring techniques used to get $k_{33} / k_{11}$ (see Ref. 10, II A) : Black points only from threshold values ${ }^{9}$, circles (our values) and shaded region ${ }^{8}$ from changing birefringence. stants $k_{33} / k_{11}$ is compared. The new measurements of SNYDER and SAUPE ${ }^{11}$ show that this ambiguity is mainly caused by uncertainties in the measured refractive indices of the nematic liquid crystal. In earlier measurements SAUPE ${ }^{9}$ found a temperature independent ratio $k_{33} / k_{11}$ from the phase difference change vs field curve. By using more accurate refractive index data to calculate the phase difference change curve, we find that $k_{33} / k_{11}$ does show some temperature dependence and new values are in good agreement with those computed only from the threshold field data. These values also agree with those calculated from the phase difference change vs field curve for the perpedicularly oriented film *.

The agreement of these various methods is reassuring and it indicates the validity of using the normal deformation to determine elastic constants. The elastic constant data to be presented in this paper were measured with a parallel oriented sample in a magnetic field. A least-squares ${ }^{12 a}$ fit to the measured birefringence at various fields was made and the two parameters of the fit were $k_{11}$ and $k_{33} / k_{11}$.

\section{II.B $k_{11}$ and $\mathbf{k}_{33}$ Measured for the 4, $\mathbf{4}^{\prime}$-Di(n-alk- oxy)-azoxybenzene Homologous Series}

Before considering the elastic constants it is worthwhile to first examine the thermal behavior for this homologous series. The familiar alternation of the nematic to isotropic phase transition temperatures is seen in Figure 2. Note that the smectic C phase makes its appearance for $n=6$ and higher values. By extrapolating the dotted line to lower $n$-values we see that the nematic region for $n=1,2$, $3 \ldots$ is quite far away from any smectic phase transition.

The temperature dependence of the splay $k_{11}$, twist $k_{22}$ and bend $k_{33}$ elastic constants are shown in Fig. 3 for the $n=1$ homolog (PAA). All elastic constants decrease with increasing temperature. Saupe defines a reduced elastic constant $C_{i i}$ as ${ }^{8,9}$

$$
C_{i i}=k_{i i} V^{7 / 3} / S^{2}
$$

* A very thin lecithin film rubbed onto the glas plates can give a weaker boundary condition which gives a lower than expected threshold condition ${ }^{12}$. With 4-methoxybenzylidene$4^{\prime}$-n-butylaniline (MBBA) we found that for the bend energy at the boundary $C=10^{-2}$ to $10^{-4} \mathrm{erg} / \mathrm{cm}^{2}$, where $C$ is defined by RapinI and Papoular ${ }^{12}$. $C$ can be much larger when less than $1 \%$ lecithin is dissolved in MBBA. 


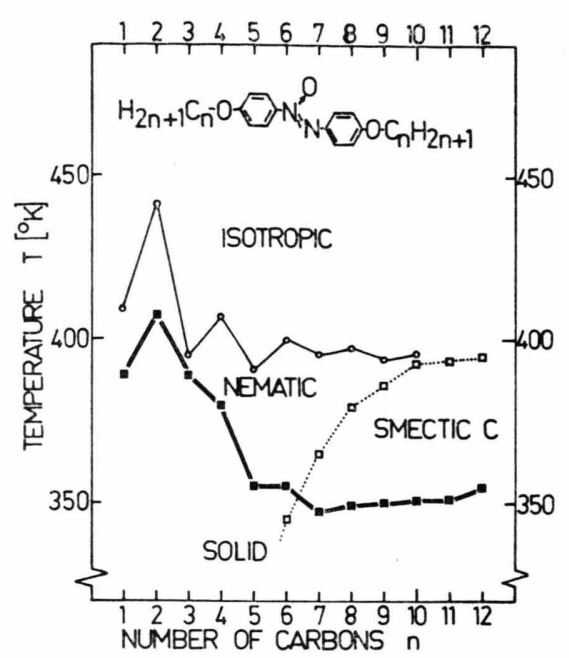

Fig. 2. Phase diagram of the $4,4^{\prime}$-di(n-alkoxy) azoxy benzene homologous series ${ }^{13}$.

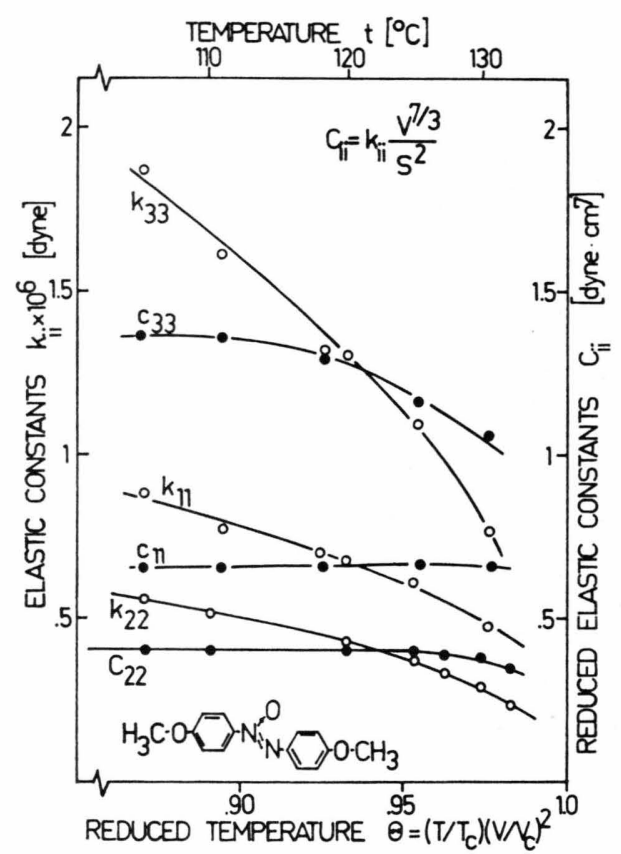

Fig. 3. The elastic constants $k_{i i}$ and the reduced elastic constants $C_{i i}(i=1,2$ and 3$)$ for the $n=1$ homolog (p-azoxyanisole) vs temperature.

where $S$ is the order parameter and $V$ is the mole volume. The temperature dependence of the long range order is contained in the $S^{2}$ factor and the Maier-Saupe theory shows that the reduced elastic constant should be independent of temperature if changes in short range order are neglected. In computing the $C_{i i}$ values for PAA we used the values of
$S$ obtained from anisotropic magnetic susceptibility data ${ }^{14}$. The mole volumes were computed from density measurements of LINSERT ${ }^{15}$.

Figure 3 shows that $C_{11}$ and $C_{22}$ are essentially independent of temperature but that $C_{33}$ decreases sharply at higher temperatures. $C_{11}$ and $C_{22}$ computed using less accurate values of the order parameter $S$ from refractive index data ${ }^{14}$ also show a small temperature dependence.

The values of the twist elastic constant $k_{22}$ were obtained from measurements of FRÉEDERICKSZ and ZWETKOFF ${ }^{16}$. We have made no measurements of $k_{22}$ for PAA or any of the higher homologs.

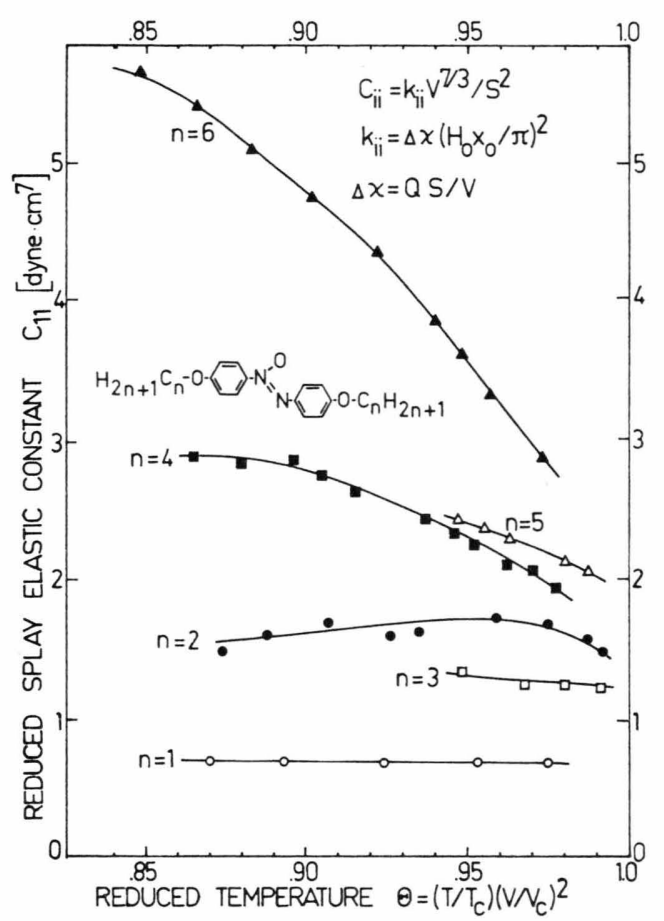

Fig. 4. The reduced splay elastic constant $C_{11}$ for the homologous series vs reduced temperature.

Figure 4 displays the reduced splay elastic constant $\mathrm{C}_{11}$ for the homologous series having $n$-values from 1 to 6 . These values were obtained from the magnetic threshold field for a parallel oriented sample (see Ref. ${ }^{10}$ ) under the assumption that the molecular diamagnetic susceptibility anisotropy is the same for all members of the series. This is a good approximation because the aromatic rings contribute most to the diamagnetic anisotropy and the length of the alkoxy-chain plays a rather small role as ZWETKOFF and SosNovsky ${ }^{17}$ have shown. 
We have also assumed that the order parameter $S$ for the entire homologous series on a reduced temperature scale is the same as for PAA. The mole volumes were already known from LINSERT's ${ }^{15}$ density measurements.

From Fig. 4 it is clear that the reduced splay elastic constant $C_{11}$ for the lower homologs $n=1,2$ and 3 is essentually temperature independent. This means that the observed temperature dependence of $k_{11}$ of these compounds is mainly a result of the temperature dependence of the order parameter $S$. For the higher homologs $n=4,5$ and 6 the reduced splay elastic constant is appreciably temperature dependent. Even taking into consideration that the order parameters for these different substances are not exactly the same on a reduced temperature scale $^{14}$ is not enough to explain this temperature dependence of $C_{\mathbf{1 1}}$. This temperature dependence of the reduced splay elastic constant $C_{11}$ then means that the structure of the short range order for the higher homologs changes with temperature since Eq. (1) was derived in the Maier-Saupe theory under the assumption that the short range order was temperature independent.

The temperature dependence of the reduced splay elastic constant is not the only hint that short range order can have an appreciable effect on the elastic constants. The reduced splay elastic constant $C_{11}$ for $n=2$ is nearly twice that for $n=1$. This is a surprising result because the molecular length has only increased by two $-\mathrm{CH}_{2}-$ groups. This large change in $C_{11}$ can be explained by assuming these two homologs have a different short range order structure. This is in agreement with mole volume measurements on these two homologs.

The mole volume as a function of the alkoxychain length for different reduced temperatures $\left(T / T_{\mathrm{c}}\right)$ is presented in Figure 5. In the nematic phase the mole volume increases about $36.3 \mathrm{~cm}^{3}$ for every $\mathrm{CH}_{2}$-group that is added to the alkoxychain. In the isotropic phase this average increase is $36.1 \mathrm{~cm}^{3}$. The actual increases are seen to deviate from these average values by about $5 \mathrm{~cm}^{3}$. The packing density therefore alternates in the nematic phase as well as in the isotropic phase. This alternation implies that the short range order must change as the alkoxy-chain length is increased even though the molecules are nearly identical. A surprising result is that lower packing densities give larger elastic constant values.

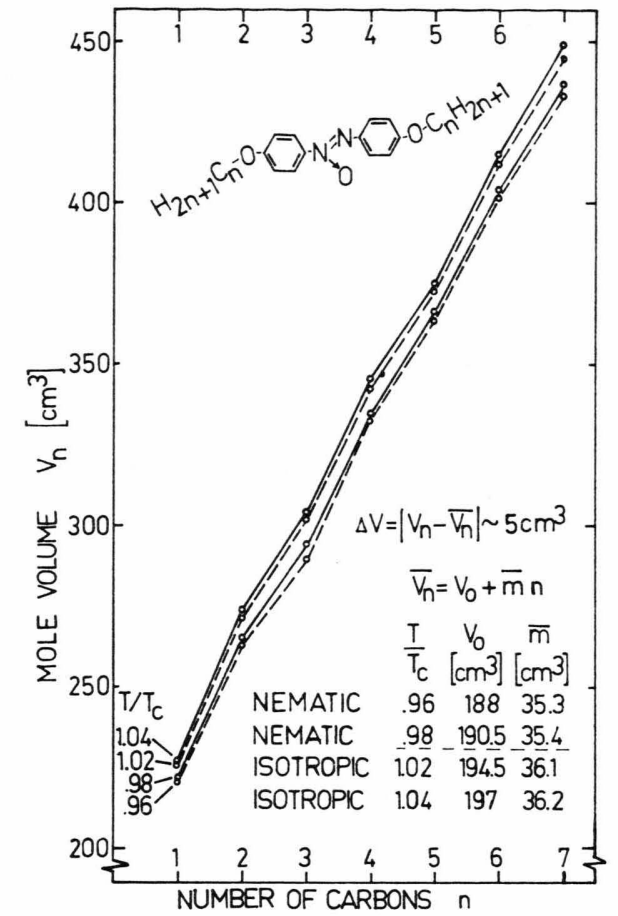

Fig. 5. The mole volume of the homologous series vs the alkyl chain length.

A further indication of changes in short range order is obtained from an examination of the sum of the reduced elastic constants. SAUPE ${ }^{9}$ has related the sum of the reduced elastic constants to two parameters $A$ and $m$, which are characteristic of each particular nematic liquid crystal.

$$
C_{11}+C_{22}+C_{33}=2.79 \cdot 10^{8} \mathrm{~A} / \mathrm{m}^{1 / 3} .
$$

$A / V^{2}$ defines the nematic potential. The number $m$ comes into the Maier-Saupe theory as a free parameter but it has a physical meaning. It is the number of the molecules in a steric unit. Turning a steric unit in a nematic takes less energy than turning a single molecule because the steric unit is nearly spherical and therefore it has little hindrance. The $A$ values for the homologous series are tabled in Ref. ${ }^{7}$. Our measurements of $C_{11}$ and $C_{33}$ combined with published values of $C_{22}{ }^{18}$ give $m_{2}=0.7 m_{1}$ for the first two homologs. This is a considerable change in short range order structure.

From Fig. 4 we see that the general trend is for the reduced elastic constants to become larger with longer alkoxy-chain length. This result is expected since longer molecules should make the nematic phase more resistant to bending and splaying ${ }^{19}$. An 
alternation is superimposed on this trend which can be quite large as is seen for the case $n=1$ and 2 . The alternation of the anisotropic polarizabilities, which explains the alternation of the nematic to isotropic transition temperature ${ }^{7}$, cannot explain this large alternation of the reduced elastic constants. Strong alternation of the reduced elastic constants can only be explained through a change in short range order.

\section{II.B1 $k_{33} / k_{11}$ Ration for the Homologous Series}

The ratio of the elastic constants $k_{33} / k_{11}$ can be determined from the magnetic field dependence of the phase difference change $\delta(H)$ by fitting a theoretical curve to the experimental data points. The ratio $k_{33} / k_{11}$ is the fitting parameter (see Ref. ${ }^{10}$ ). Figure 6 shows these measurements as a function of

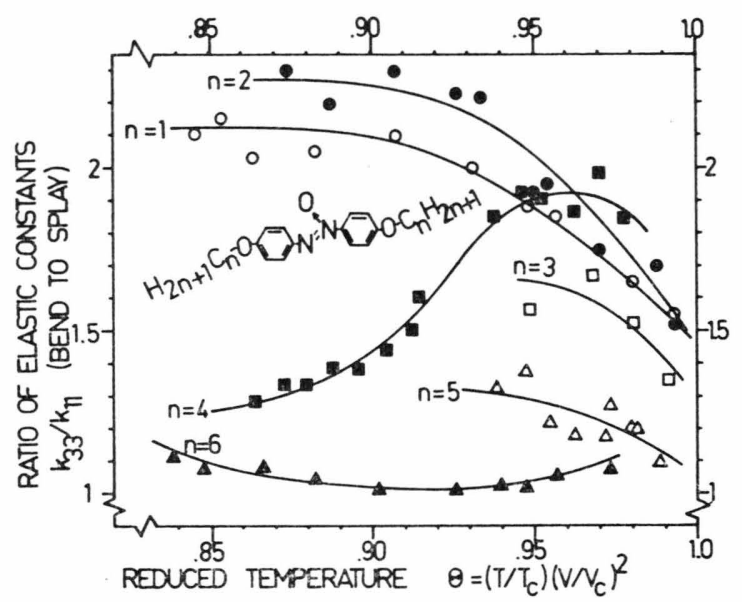

Fig. 6. The ratio $k_{33} / k_{11}$ of the homologous series vs reduced temperature.

temperature for the homologous series. If the short range order is not affected by temperature the MaierSaupe theory indicates that the $k_{33} / k_{11}$ ratio should be a constant ${ }^{8}$. For the homologs with $n=1$ and 2 at lower temperatures, the ratio of the bend to splay elastic constants is larger than 2 and temperature independent. From a mean field model which neglects all short range order effects NEHRING and SAUPE ${ }^{20}$ have calculated that the bend elastic constant should equal the splay elastic constant.

At higher temperature for $n=1$ and $2, k_{33} / k_{11}$ is no longer temperature independent. The ratio of the elastic constants monotonically decreases to unity with increasing temperature. The temperature behavior of $k_{33} / k_{11}$ in this region must be due to structural changes in short range order. For the $n=4$ homolog, the ratio of the elastic constants shows a very unusual temperature behaviour which we believe is caused by a planar-nonplanar transition of the short range order structure (see Section II.C). For the $n=6$ homolog at lower temperatures $k_{33} / k_{11}$ weakly decreases.

\section{II.C Discussion of Experimental Results}

The elastic constants of this series show a remarkable temperature dependence as can be seen in Figures 4-7. We will attempt to explain this through temperature changes in long and short range order. We will discuss changes in short range order from a phenomenological standpoint in order to give it a more physical meaning. In practice it is possible to determine short range structure in a nematic liquid crystal from X-ray scattering experiments ${ }^{21,22}$.

In liquids, there exists an interaction potential between the molecules which leads to a characteristic short range order structure. The nature of this exchange energy can be due to e. g. steric hinderance, dipole-dipole interaction, and so on. The Boltzmann factor (interaction energy/kT) determines which interaction will be dominant for any given temperature. The Maier-Saupe theory is valid in the nematic phase of the $n=1$ homolog, and this indicates that the dominant interaction energy between the molecules is due to induced dipole-induced dipole or Van der Waals forces ${ }^{7}$. For elongated molecules the polarizability in the direction of the long molecular axis is larger than the polarizability perpendicular to this molecular axis. Under these conditions the interaction energy is maximum when the molecules lie end to end and minimum when the molecules lie side by side. This type of exchange energy leads to a molecular distribution where the centers of mass of the molecules are irregularly distributed ${ }^{7}$.

There are other intermolecular forces that tend to make the molecules lie next to each other so that the molecular centers lie on a straight line. At lower temperatures these forces produce a smectic phase. The centers of the molecules are no longer irregularly spaced as for the case of the simple Van der Waals forces and therefore the short range order is quite different for these two cases. 
The short range order expected for these forces is schematically illustrated in Figure 7 . In region $I$ and $I I$ we have sketched a short range order structure resulting from only Van der Waals forces and the molecular centers are irregularely distributed. The short range order structure in region I changes very strongly with temperature and this can be interpreted as a pretransitional effect to the nearby isotropic phase.

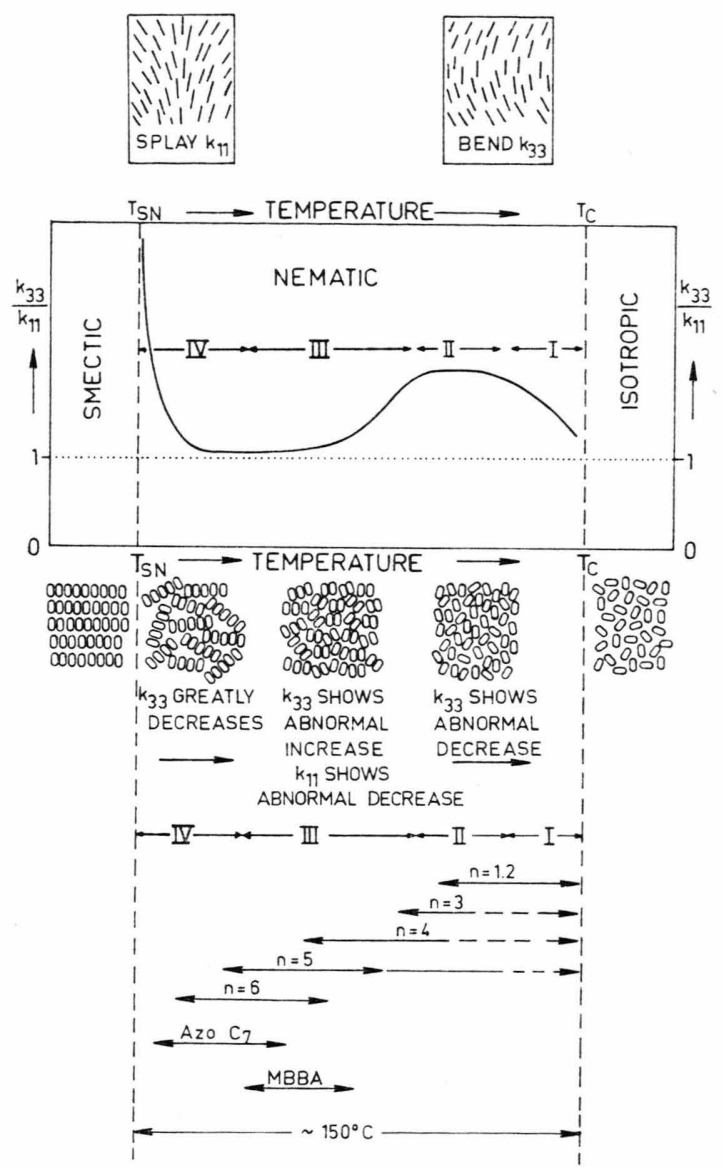

Fig. 7. A schmeatic picture of $k_{33} / k_{11}$ as a function of short range order structure deduced from the elastic constant measurements of the entire homologous series.

In region $I I I$ forces that tend to bring the molecules into groups come into play. These groups represent the pretransitional beginning of the smectic phase. This molecular association is shown as pairbuilding in Figure 7. A few degrees above the nematic to smectic phase transition these association forces dominate and build up large cybotactic clusters of molecules which possess a structure similar to that of the smectic phase. These cybotactic groups occur in region IV.

Now let us try to explain the temperature dependence of the elastic constants of the homologous series through changes in short range order.

Figure 7 summarizes the abnormal temperature behavior of the $k_{33} / k_{11}$ ratio for these compounds. We treat only the ratio of the elastic constants here because this eliminates the temperature dependence which simply enters via the temperature dependence of the long range order parameter $S$. A summary of the temperature dependence of the reduced elastic constants $C_{i i}$ is given by GRULER and MEIER ${ }^{23}$.

This curve was built piece-wise from the elastic constant measurements of the homologous series. The behavior for $n=1$ and 2, for example, corresponds to region I and II. We could not measure the elastic constants of these materials which would correspond to the region III, because they crystallized above this temperature. On this same curve the measurements for $n=4$ extend from region I to region III, but the regions I and II are not so pronounced. For the $n=5$ and 6 homologs regions I and II were not observed at all. The special changes of the short range order structure which is characterized by regions I and II are probably compressed into a very small temperature region just before the nematic - isotropic transition.

This curve schematically represents a continuous change in structure of short range order. The long range order does not directly depend upon the short range order and the nematic-isotropic phase transition can occur in any region. The cybotactic groups of the (nematic) region IV, for example, still exist at the nematic-isotropic phase transition as $\mathrm{DE}$ VRIES ${ }^{21}$ has shown. In this phenomenological picture of the short range order structure the existence of the smectic phase plays the most important role. The nematic-isotropic phase transition temperature is only used to estimate the long range order parameter $S$. It should be possible to determine the short range order structure of regions I-IV with $x$-ray scattering experiments ${ }^{21,22}$. The data from $\mathrm{x}$-ray scattering are not complete for the series, so at the moment we are forced to use our less rigorous phenomenological picture. In the following we will discuss each region separately.

Region IV: The pretransitional effect in the elastic constants is well explained by the existence 


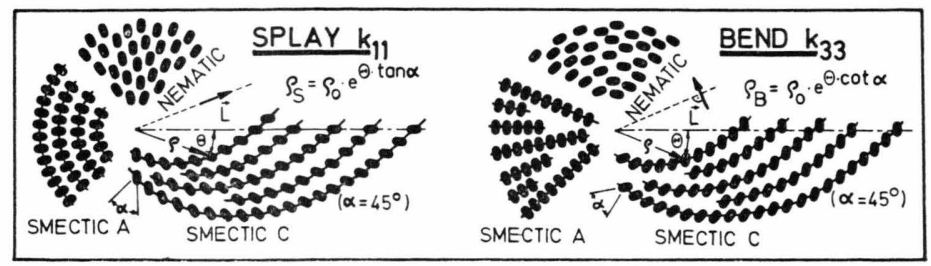

Fig. 8. A pure splay and bend deformation for the nematic smectic $\mathrm{A}$, and smectic $\mathrm{C}$.

of the large cybotactic groups having a planar, smectic-like structure. According to DE VRIES ${ }^{21}$, these cybotactic groups contain at least 100 or so molecules. It is easier to understand the temperature dependene of the reduced splay $C_{11}$ and bend $C_{33}$ elastic constants in region IV if we first discuss the magnitude of splay and bend elastic constants in the smectic phase.

Figure 8 illustrates a pure bend and splay deformation in a nematic, smectic A and smectic C phase. (The singularity can be ignored for this description.) The parallel smectic planes, where the director $\boldsymbol{L}$ makes a fixed angle $\alpha$ to the plane, are bent into the form of a logarithmic spiral. The bend and splay deformations are contrary to the smectic structure because the distance between two planes is a function of position. It is only possible to have bend and splay deformations in a smectic $C$ phase $(\alpha \neq 0)$ if the planes are broken as is illustrated in Figure 8. This would require a lot of energy and therefore $k_{11}$ and $k_{33}$ must be very large in the smectic C phase. For the case $\alpha=0$, which holds for the smectic A phase, a splay deformation is not contrary to the planar structure but a bend deformation is only possible by breaking the smectic planes (Figure 8). For a smectic A phase we would expect $k_{33}$ to have a very large value and $k_{11}$ to be comparable with the value in the nematic phase.

The smectic-like cybotactic groups carry the elastic properties of the smectic phase over into the nemactic phase as a pretransitional effect. Since DE VRIES ${ }^{21}$ found cybotactic groups having a structure similar to the smectic $\mathrm{A}$ and $\mathrm{C}$ phases, we expect also a different behavior in the elastic constants of a nematic phase that has a lower smectic A or C phase.

Cheung and Meyer ${ }^{24}$ found only a strong increase in $k_{33}$ at the nematic to smectic A phase transition. They observed no strong abnormalities in the splay elastic constant $k_{11}$. These observations are in agreement with our predictions. A smectic C phase exists for the higher homologs of the $4,4^{\prime}$ - di(n-alkoxy) azoxybenzenes. The reduced splay elastic constant $C_{11}$ and the reduced bend elastic constant $\mathrm{C}_{33}$ show a strong increase near the smectic C phase for the $n=6$ homolog.

Near the smectic $C$ phase the ratio of the elastic constants $k_{33} / k_{11}=C_{33} / C_{11}$ of the homologous series is nearly temperature independent. This can be explained if $\alpha$ is around $45^{\circ}$. The $4,4^{\prime}$-di (n-heptyloxy) azobenzene compound (Figure 9), however,
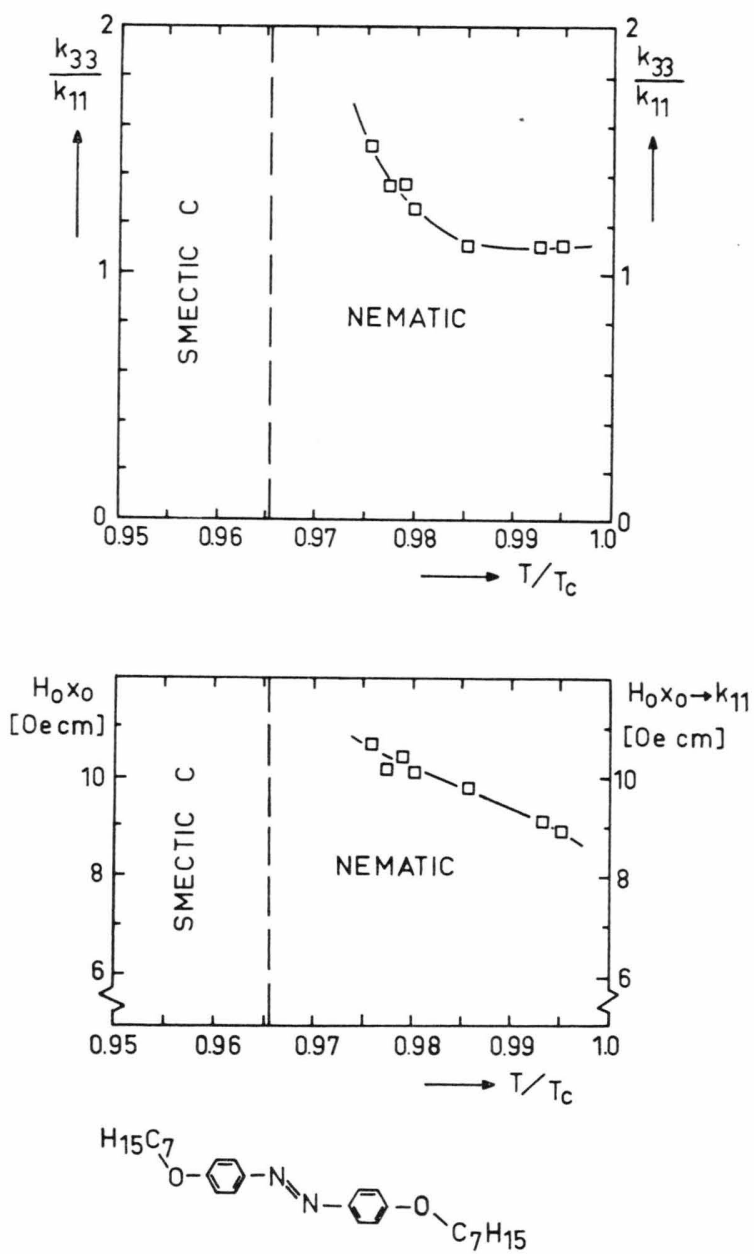

Fig. 9. (upper) $k_{33} / k_{11}$ of the $4,4^{\prime}-$ di (n-heptyloxy) azobenzene compound vs $T / T_{\mathrm{c}}$. (lower) threshold value $H_{0} x_{0}=$ $\pi\left(k_{11} / \Delta \chi\right)^{1 / 2}$ vs $T / / T_{\mathrm{c}}$. 
which also has a smectic $\mathrm{C}$ phase, shows an increase in $k_{33} / k_{11}$ with temperature. This means that $\alpha$ must be smaller than $45^{\circ}$.

Region III: The cybotactic groups become smaller a few degrees above the smectic to nematic phase transition temperature. When they contain only a few molecules, e.g. two to four, then we can no longer discusse a planar structure in terms of cybotactic groups. From x-ray scattering experiments, Chistyakov and Chaikowsky ${ }^{22}$ found a planar short range order structure which we interpret as a planar arrangement of steric units. Since they made the measurements on the same homologous series it will be useful to review their results: They saw no indication of a planar structure in the homologs $\mathrm{n}=1$ and 3 . The homologs $n=2$ and 4 to 10 , however, did show more or less strongly the existence of a planar short range order structure. This does not contradict the phenomenological picture where region II was defined by Van der Waals forces.

Region I and II: Chistyakov and Chaikowsky found a non-planar short range structure in region I and II. Region I is seperated from region II because the thermal expansion coefficient shows an abnormal temperature behavior in region $\mathrm{I}^{7,15}$.

The changes of the reduced elastic constants from region II to III can be explained by change from a non-planar to a planar short range order structure as will be shown in the nect section.

We believe these changes in the structure of the short range order are not specific to this particular homologous series but are quite general. Because of the strong connection between short range order structure and elastic constants we expect that the elastic constants of other homologous series will show similar behavior. Let us look for example at MBBA where the elastic constants can be characterized as falling in region III ${ }^{25}$. The ratio of the measured elastic constants shows a similar temperature dependence as the $n=4$ homolog, but it is not so pronounced. The short range order structure belongs mainly to the region III. As far as we know MBBA has no smectic phase. However, if we increase the length of the molecule by substituting a $\mathrm{C}_{3} \mathrm{H}_{7}-\mathrm{O}$ - group instead of the $\mathrm{CH}_{3}-\mathrm{O}$ - terminal group then the new compound does have a smectic C phase ${ }^{26}$. MBBA can be compared to the homologs where $n=4$ and 5 and which occur in region III.
It seems to us that it is possible to decrease the forces which will form a smectic phase by adding a foreign substance to the nematic phase. HALLER ${ }^{25}$ and the author found for pure MBBA that the ratio $k_{33} / k_{11}=1.3$ is independent of temperature if the reduced temperature is far enough from the nematic to isotropic transition $\left(T_{\mathrm{c}} \sim 42{ }^{\circ} \mathrm{C}\right)$. A mixture of MBBA and $7.9 \%$ of a cyano-compound $\left(T_{\mathrm{c}} \sim 36^{\circ} \mathrm{C}\right)$ gave an elastic constant ratio $k_{33} / \mathrm{k}_{11}=1.7^{10}$. This large difference in the ratios can not be explained through the larger value $k_{33} / k_{11}$ which the cyanocompound has. Therefore, it seems reasonable that the short range order structure has changed. In order to obtain the absolute value of the elastic constant we assume that the diamagnetic susceptibility anisotropy of the mixture is equal to or a few percent smaller than that for pure MBBA. We find that at the same reduced temperature the bend elastic constant $k_{33}$ has nearly the same value as that for pure MBBA. The splay elastic constant $k_{11}$, however, has a larger value for pure MBBA than it has for the mixture. The change of the elastic constants of the mixture is in agreement with our picture which we have developed from the $4,4^{\prime}$-di (nalkoxy) azoxybenzene series.

\section{Model Calculation for the Elastic Constants of a Nematic Liquid Crystal}

SAUPE 9 calculated the elastic constants of a nematic liquid crystal from the interaction potential of the Maier-Saupe theory. For this calculation he assumed an an isotropic distribution of the molecular centers. He obtained

$$
C_{11}: C_{22}: C_{33}=k_{11}: k_{22}: k_{33}=-7: 11: 17 \text {. }
$$

The two elastic constants $k_{22}$ and $k_{33}$ are positive, as would be expected, but $k_{11}$ is negative. A negative value of $k_{11}$ would seem to indicate that a uniformly oriented nematic phase would not be the most stable equilibrium structure. Saupe carried through a different model calculation and found that all three elastic constants are positive. For this calculation he assumed a short range order structure that was a simple cubic lattice where a four-fold symmetry axis corresponds to the local optical axis.

Chandrasekar, Madhusudana, and Shubha ${ }^{27}$ extended the Maier-Saupe theory by including dipole-dipole, induced-dipole-induced dipole and other forces. They calculated the elastic constants from 
this type of exchange energy by assuming a simple cubic lattice for the calculation of the twist elastic constant $k_{22}$ and compared the value with the $n=1$ and 2 homologs. The theoretical value is in good agreement with the experiment.

NEHRING and SAUPE ${ }^{20}$ calculated the ratio of the elastic constants in a more rigorous form than Saupe's earlier treatment and found

$$
C_{11}: C_{22}: C_{33}=k_{11}: k_{22}: k_{33}=5: 11: 5 \text {. }
$$

This calculation still uses the basic Maier-Saupe assumption of an isotropic distribution of the molecular centers.

PRIEST ${ }^{28}$ calculated the elastic constants from a lattice model. He found that

$$
\frac{k_{33}}{k_{11}}=\frac{1}{2} \sum_{i}^{\sum_{i} A\left(\boldsymbol{r}_{\mathrm{oi}}\right) \varrho_{\mathrm{oi}}{ }^{2}}
$$

where $A\left(\boldsymbol{r}_{\mathrm{oi}}\right)$ is the exchange energy potential. $\boldsymbol{r}_{\mathrm{oi}}$ is the distance between the $\mathrm{i}$-th and the o-th molecule, and $\varrho_{0 \mathrm{i}}=\boldsymbol{r}_{\mathrm{oi}} \times \mathbf{L}$ and $z_{0 \mathrm{i}}$ is the $z$-component of $\boldsymbol{r}_{\mathrm{oi}}$. We assume the following functional form for $A$ $A\left(\boldsymbol{r}_{\mathrm{oi}}\right)=A_{1} / r_{\mathrm{oi}}{ }^{6}\left[1+\left(A_{2} / A_{1}-1\right)\right] \varrho_{\mathrm{oi}}{ }^{2}\left(\varrho_{\mathrm{oi}}{ }^{2}+z_{\mathrm{oi}}{ }^{2}\right)^{-1}$. We do not need to specify the parameters $A_{1}$ and $A_{2}$ for the potential. In the following we assume that $A_{2} / A_{1}=0.5 \mathrm{Eq}$. (3) can then be written as

$$
k_{33} / k_{11}=\left(a_{33} / a_{11}\right)(b / l)^{4}
$$

where $a_{33}$ and $a_{11}$ are structure factors. $l$ and $b$ describe the size of the steric unit.

First we assume that the steric units are arranged in a hexagonal planar array. This could be a possible short range order structure for the region III. If we assume that the steric unit is equal to the

\begin{tabular}{|c|c|c|c|c|}
\hline Structure & & Planar & & Non \\
\hline$l / b$ & 3 & 1.5 & 1.0 & 1.5 \\
\hline$k_{33} / k_{11}$ & 0.019 & 0.2 & 0.35 & 1.2 \\
\hline$a_{33}$ & 11 & 6 & 3.5 & 32 \\
\hline$a_{11}$ & 4 & 7 & i0 & 6 \\
\hline
\end{tabular}
molecular size, than we can see from Table I that

Table 1.

the ratio of the elastic constants $k_{33} / k_{11}$ is too small. By changing the form of the steric unit into a sphere we get nearly the measured value. If we assume that the steric units are arranged in a nonplanar structure, as is shown in Figure 10, we get quite a different value for the ratio of the elastic constants (Tab. 1). The difference $\Delta\left(k_{33} / k_{11}\right)$ between $k_{33} / k_{11}$ obtained from a non-planar structure and $k_{33} / k_{11}$ from regions II and III.

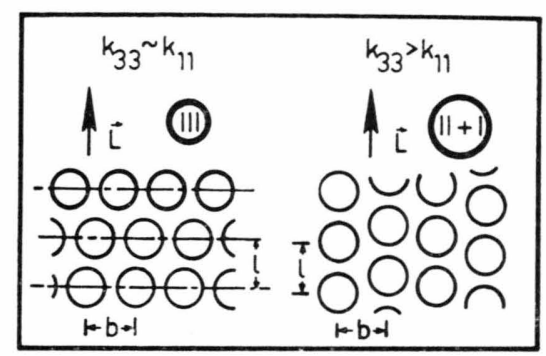

Fig. 10. Schematic representation of possible arrangements of steric units in the nematic regions III and II + I.

Furthermore, these model calculations show that the structural factor $a_{33}$ makes larger changes than $a_{11}$ when the structure of the short range order is changed. This could be a reason why $C_{33}$ is much more strongly temperature dependent in region I then $C_{11}$ is.

Since the longer molecules should have larger sterical units, it is possible to explain the increase of the reduced elastic constants with increasing alkoxy chain length with the lattice model. Neglecting structural changes from Priest's lattice model we get

$$
C_{11} \sim l^{2} \quad \text { and } \quad C_{33} \sim b^{2} .
$$

If the steric unit is proportional to the molecular length we get an increase of the reduced elastic constants with increasing alkoxy chain length. This explains the average effect, but not the large observed alternation. A large discrepancy is seen for the $n=6$ homolog. This is not surprising because for this homolog we know from other data that cybotactic groups exist which are much larger than the steric units.

\section{Acknowledgements}

The author is grateful to Dr. A. SAupe for fruitful discussions where he pointed out the necessity for more elastic constant data. I also would like to thank Dr. Tery J. SCheffer for stimulating and helpful discussions. The author is indepted to Dr. G. MEIER for his support in this work. I also wish to thank Dr. H. DEULING for the least squares fitting program. 
1 A good article reviewing earlier papers is given C. W. OSEen, Trans. Faraday Soc. 29, 883 [1933].

2 A good article reviewing earlier papers is given H. ZOCHER, Trans. Faraday Soc. 29, 945 [1933].

3 F. C. Frank, Disc. Faraday Soc. 25, 19 [1958].

4 J. Nehring and A. SAupe, J. Chem. Phys. 54, 337 [1971].

5 F. JÄhnig and H. Schmidt, Ann. Phys. 71, 129 [1972].

${ }^{6}$ P. C. Martin, O. Parodi, and P. S. Pershan, A unified hydrodynamic theory for crystals, liquid crystals, and normal fluids, submitted to Phys. Rev.

7 W. Maier and A. SAupe, Z. Naturforsch. 14 a, 882 [1959] ; 15 a, 287 [1960].

8 A. SAUPE, Z. Naturforsch. 15 a, 810 [1960].

9 A. SaUpe, Z. Naturforsch. 15 a, 815 [1960].

10 H. Gruler, T. J. Scheffer, and G. Meier, Z. Naturforsch. 27 a, 966 [1972].

11 W. SNydeR and A. SAupe, IV. International Liquid Crystal Conference in Kent, Ohio 1972.

12 A. Rapini and M. Papoular, J. Physique 30, C4-54 [1969].

${ }^{12 a}$ Copies of the program in Fortran IV are available from Dr. H. Deuling, Freie Universität Berlin, D-1000 Berlin 33, Arminallee 3.

13 C. H. Brown and W. G. Schaw, Chem. Rev. 57, 1049 [1957].

14 Order parameter data are summarized in A. SAUPE and W. Maier, Z. Naturforsch. 16a, 816 [1961]; see also A. SAupe, Angew. Chem. internat. Edit. 7, 97 [1968].
15 F. LinSERT, Diplomarbeit 1945, Universität Halle.

16 V. FréedericksZ and V. Zwetkoff, Phys. Z. Sowjetunion 6, 490 [1934].

17 V. Zwetkoff and A. Sosnovsky, Acta Physicochim. URSS 18, 358 [1943].

18 Orsay Liquid Crystal Group in "Liquid Crystals and Ordered Fluids", Editor J. F. Johnson and R. S. Porter, Plenum Press, New York-London 1970, p. 447.

19 I. C. Lubensky, Phys. Lett. 34 A, 202 [1970].

20 J. Nehring and A. Saupe, J. Chem. Phys. 56, 5527 [1972].

21 A. De Vries, Mol. Cryst. and Liq. Cryst. 10, 31, 219 [1970].

22 I. G. Chistyakov and W. M. Chaikowsky, Mol. Cryst. and Liq. Cryst. 7, 269 [1969].

${ }^{23}$ H. Gruler and G. Meier, submitted to Mol. Cryst. and Liq. Cryst., in press.

24 L. Cheung and R. B. Meyer, IV. International Liquid Crystal Conference in Kent, Ohio 1972.

25 I. Haller, J. Chem. Phys. 57, 1400 [1972].

26 G. W. SMith, Z. G. GARdLund, and R. J. CuRTis, IV. International Liquid Crystal Conference in Kent, Ohio 1972.

27 S. Chandrasekar, N. V. Madhusudana, and K. Shubha, Acta Crystallogr. Sect. A 1972, 28.

28 R. G. Priest, Mol. Cryst. and Liq. Cryst. 17, 129 [1972]. 